\title{
SIMILARITIES AND DIFFERENCES BETWEEN MAGNETIC AND NON-MAGNETIC CP STARS
}

\author{
Thierry LANZ *) \\ NASA Goddard Space Flight Center, Code 681, \\ Greenbelt, MD 20771, USA
}

\begin{abstract}
This brief review discusses the similarities between CP stars, focusing on magnetic fields and chemical abundances. New evidence for the presence of a magnetic field with a complex structure in some CP1 stars is presented. Small-scale structures on the surface of these stars may be connected with such a field, and the resulting effects on the curve-ofgrowth have been investigated. Finally, the changes during evolutionary time-scales on surface abundances are advocated as one possible reason of the large observed scatter in the chemical anomalies inside given groups of CP stars.
\end{abstract}

\section{INTRODUCTION}

Since the review of Preston(1974) on the chemically peculiar stars of the upper main sequence (CP stars), one recognizes two different sequences of CP stars in the temperature range $7000 \mathrm{~K}$ to $15000 \mathrm{~K}$. The Ap stars with $\mathrm{Sr}, \mathrm{Cr}, \mathrm{Eu}$, or $\mathrm{Si}$, peculiarities (CP2 stars) are distinguished from the Am stars (CP1 stars) and the Ap-Bp HgMn stars (CP3 stars) by the fact that a significant fraction of the former have been observed to possess a magnetic field with a large-scale structure, while the latter do not show such a field. Preston (1974) regrouped the stars with helium peculiarities in the CP4 group (He weak stars), but later on the heterogeneous composition of this group was pointed out. Some CP4 stars are related to the CP3 stars with strong spectral lines of P II, Ga II and Y II, while the other CP4 stars are connected with the CP2 stars with strong Si II lines and an observed magnetic field. These CP4 stars are generally hotter $(13000$ to $18000 \mathrm{~K})$ and appear to extend the two sequences of magnetic and non-magnetic CP stars. To complete this picture of the chemically peculiar stars of the upper main-sequence, one ought to mention the metal-weak $\lambda$ Bootis stars and the He-rich early B stars (Wolff 1983). The general properties of these stars have been described by Preston (1974), Wolff (1983) and in several reviews during this and recent past meetings (see e.g. Adelman \& Cowley 1986, Cayrel et al. 1991, Takada-Hidai 1991, Ryabchikova 1991).

*) NRC-NASA Research Associate 
This overall picture of CP stars is based at first on the empirical spectral classification work. However, it is surely indicative of the basic physical processes at work in their atmospheres. In particular, it links some chemical anomalies to the stellar magnetic field. Despite its usefulness to illuminate basic processes, such a classification may hinder a more complete understanding overlooking peculiar cases, like e.g. stars classified as Ap HgMnSi. Moreover, the difficulty to find bona fide normal $A$ stars as soon as high-quality observations are available (if a "normal" star is assumed to be a solar-composition star) suggests that there is a continuous smooth transition from the "normal" to the chemically peculiar stars. Finally, the groups of CP stars are apparently well-characterized, but stars with large chemical abundance differences may be assembled in a same group. For example, Takada-Hidai (1991) showed for the CP3 stars a large scatter for all observed species.

That is the reasons why we are aiming to address the question of the similarities and the differences between CP stars to assess more closely this overall picture. First we must discuss the grounds to distinguish the two sequences of magnetic and non-magnetic stars: do all CP2 stars possess a magnetic field? on which basis are CP1 and CP3 stars regarded as non-magnetic? Our second purpose is to tackle the problem of the very large differences in abundance anomalies to see which additional insights the scatter itself may give us.

\section{MAGNETIC FIELDS IN CP STARS}

Two recent reviews discussed the observations of magnetic fields in CP stars (Mathys 1989, Landstreet 1992). Stellar magnetic fields are diagnosed via the Zeeman effect through its two properties: energy level splitting and polarization. The splitting of the spectral lines can be observed in the stellar spectra if the apparent rotational stellar velocity is low enough and the magnetic field sufficiently strong. It is the most direct and the most easily interpreted way to detect a magnetic field in a star. As the splitting increases linearly with the Landé factor of the spectral line and quadratically with the wavelength, an appropriate choice of the spectral interval will influence the detection limit. Mathys (1990) showed that the Fe II $\lambda 6149.2$ line is particularly adequate, and found 4 new CP stars with resolved magnetically split lines. Subsequently, Mathys \& Lanz (1992) and Mathys etal. (these proceedings) reported the discovery of 12 other CP stars with resolved spectral lines. The separation of the Zeeman components directly gives the magnetic field modulus, averaged over the visible stellar hemisphere. To date 28 stars are known to show such resolved lines, and all are CP2 stars. The lowest measured field is of the order of $3 \mathrm{kG}$, setting a typical limit to resolve magnetically split lines even for stars with a very low apparent rotational velocity. However, the Zeeman effect would still somewhat broaden the spectral lines for weak magnetic fields. In this case, the detection limit may be lowered to about $1 \mathrm{kG}$; for weaker fields, the thermal broadening becomes then greater than the Zeeman broadening. Preston (1971) used this magnetic broadening to estimate the mean magnetic field modulus in several very sharp-lined CP2 stars.

The polarization properties of the Zeeman effect furnish a second way to diagnose a stellar magnetic field. The difference between the left and the right circular polarization in the line wings is approximately proportional to the mean longitudinal field. Most measurements have been recorded in the $\mathrm{H} \beta$ line. This 
line is very broad, which removes the strong limitation due to the rotational broadening. On the other hand, a field can be detected only if it possess a large-scale structure! Recently, Mathys (these proceedings) developed an original moment technique applied to the shapes of metallic lines recorded in both circular polarizations, which allows him to derive more informations on the field structure. These techniques use differential measurements; thus, it is possible to detect weak fields, even if the difference between the two polarizations is quite small. The typical uncertainties are 100 to $300 \mathrm{G}$, but in a few cases formal errors below $50 \mathrm{G}$ were indeed achieved. Therefore, these methods are clearly more powerful than the methods based on Zeeman splitting or broadening in order to detect a magnetic field as long as the magnetic field is structured on a large scale.

The presence of magnetic fields in CP2 stars was firmly established already long ago (Babcock 1947, 1958) and has been supported by subsequent studies. But, we may ask if all CP2 stars actually possess a field. A quick look to the literature suggests that the CP2 stars have strong fields, but only a few percents of them have indeed a field strength larger than $1 \mathrm{kG}$ (Landstreet 1992). The stars with strong fields have been studied mostly, biasing our insight (this bias is furthermore increased by the works on spectral line broadening or splitting). The observed field strengths $\left\langle B_{l}^{2}\right\rangle^{1 / 2}$ range from $100 \mathrm{G}$ up to $16 \mathrm{kG}$, with a distribution strongly skewed towards small field values (Landstreet 1992). This implies that the CP2 stars with unsuccessful attempts to measure a field probably have a weak field below $300 \mathrm{G}$. A dogged work might lead to an actual detection, as illustrated by the case of $\epsilon$ UMa which possess a magnetic field with extrema of about +128 and $-64 \mathrm{G}$ (Bohlender \& Landstreet 1990). Nevertheless many fairly bright CP2 stars should still have a field which is below the current threshold of detection. By comparison, the absence of magnetic field in normal stars has been very well assessed by Landstreet (1982), who did not find a field in any out of 3109.5 - F6 stars with a median error of about $65 \mathrm{G}$.

\section{A search for magnetic fields in CP1 and CP3 stars}

Conti (1969), Borra \& Landstreet (1980) and Landstreet (1982) did not find evidence of longitudinal magnetic fields in CP1 and CP 3 stars with limits around 100 to $200 \mathrm{G}$. It therefore means that these stars have not at least an organized magnetic field like the CP2 stars. However, many elemental abundance studies in CP1 stars required to introduce a high microturbulence. This may at least in part mimic other broadening mechanisms like the Zeeman effect. Moreover, several claims of (periodic) spectral variations in CP1 stars - which is a typical characteristics of magnetic CP2 stars - have been reported (see e.g. Rao et al. 1990). These clues, as well as the fundamental question about the relation between the chemical anomalies and the stellar magnetic field, suggested to look for a magnetic field having a complex structure. It can then be detected only from the Zeeman broadening of the spectral lines.

Mathys (1988) found a magnetic field of about $2 \mathrm{kG}$ in the hot Am star - Peg from the Stenflo-Lindegren multi-line technique, using the differential broadening of lines having different Zeeman sensitivities. Mathys \& Lanz (1990) supported this claim with additional high $\mathrm{S} / \mathrm{N}$ ratio observations of a pair of $\mathrm{Fe}$ II lines of the same multiplet at 6147.7 and $6149.2 \AA$. In non-magnetic stars, these two lines have exactly the same strength, but they have a different Zeeman pattern and they are then differently sensitive to saturation in presence of a 


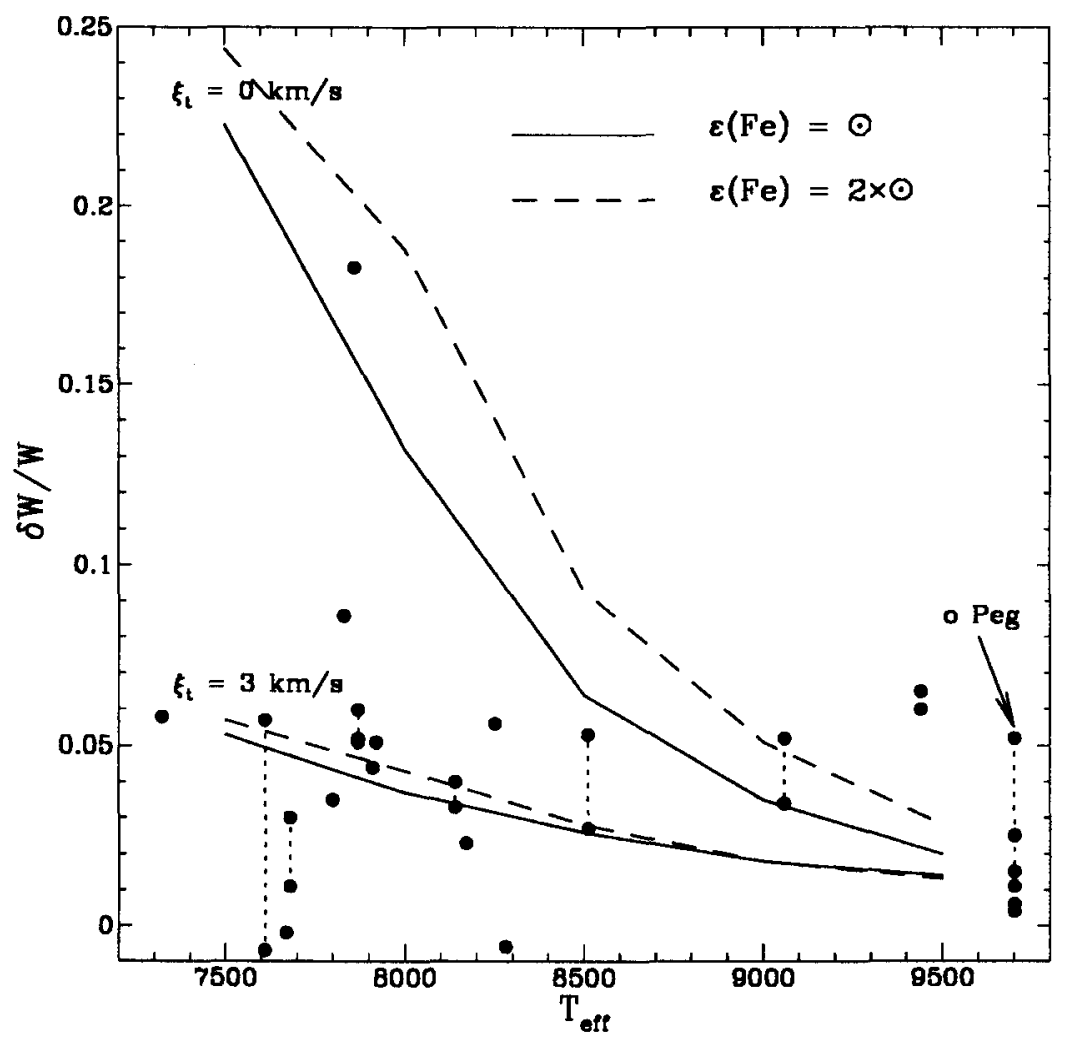

Fig. 1. Relative intensification of the Fe II lines at 6147.7 and $6149.2 \AA$ for different stellar parameters (lines), and observations in Am stars (dots). Observations for the same star are connected by dotted lines to show the variation ranges.

magnetic field. Mathys \& Lanz (1990) found a relative intensification $\delta W / \bar{W}$ of about $5 \%$ in o Peg, while it is less than $2 \%$ in non-magnetic stars and more then $13 \%$ in stars with resolved magnetically split lines having a field larger than $3 \mathrm{kG}$. The two methods therefore give a compelling evidence of the presence in o Peg of a magnetic field of about $2 \mathrm{kG}$ and having a complex structure. Takeda (1991) recently completed a detailed theoretical study of the relative intensification of this pair of lines; he proposed a second suitable pair of $\mathrm{Fe} I I$ lines in the blue spectrum for searching magnetic fields in these stars.

Lanz \& Mathys (1992) are extending this search to other CP1 and CP3 stars. They observed about 20 Am stars in October 1990 from the Observatoire de Haute-Provence (France) with the spectrometer Aurélie attached to the $1.5 \mathrm{~m}$ telescope in a range centered around $6150 \AA$. A resolution of $0.1 \AA$ and $S / N$ ratios greater than 100 were achieved. Fig. 1 summarizes their preliminary results. It displays the relative difference between the equivalent widths of the two Fe II $\lambda \lambda 6147.7,6149.2$ lines. The first Fe II line is blended by the Fe I $\lambda 6147.8$ line, whose contribution increases at lower temperature; the second Fe II line is not 
blended. We show in Fig. 1 the LTE predictions, including the blending Fe I line, for different effective temperatures, iron abundances, microturbulences, and assuming the surface gravity $\log g=4$. The presence of a magnetic field could be asserted only if the relative difference of the equivalent widths is greater than the largest prediction without magnetic intensification. Three Am stars besides - Peg seem to be good candidates to possess a magnetic field. Presently, we are assessing this result with new observations covering a large wavelength range to apply a multi-line technique. We got also 5 more observations of o Peg; the relative intensification is smaller and it might suggest spectral variations. In a few other cases, spectral variations seem definitely present. Finally, one ought to mention a negative result for Sirius, for which we got $\delta W / \bar{W} \approx 1 \%$. TakadaHidai \& Jugaku (these proceedings) give a similar result. In our opinion, we cannot assert the presence of a magnetic field with such a small value (though a field strength may be deduced from Takeda's (1991) relation), and a definite conclusion could be reached only from a multi-line approach. A detailed account of these new observations will be published elsewhere (Lanz \& Mathys 1992).

These new results for o Peg and several other CP1 stars raised up the interest to look for magnetic fields with complex structure also in the "non-magnetic" CP3 stars. However, Takada-Hidai \& Jugaku (1992) showed convincingly that the Fe II $\lambda 6149.2$ line is blended by the $\mathrm{Hg}$ II $\lambda 6149.5$. The contribution of this $\mathrm{Hg}$ II line is negligible when the mercury abundance is about solar, but this blend prevents the use of this pair of $\mathrm{Fe}$ II lines to diagnose a magnetic field in mercury-rich stars like $\mu$ Lep $([\mathrm{Hg} / \mathrm{H}] \approx+5.2$, Takada-Hidai \& Jugaku 1992). Observations by Lanz \& Mathys (1992) for 4 CP 3 stars also show a stronger feature at $\lambda 6149.2$ and support the conclusion of Takada-Hidai \& Jugaku (1992). The study of the CP3 stars deserves a new effort and it should therefore be carried out with a multi-line technique to settle this question. One ought nevertheless to mention that recent elemental abundance studies of CP3 stars (e.g. Adelman 1988a) give low microturbulences, contrary to the case of the CP1 stars, and they cannot be regarded as an indication of magnetic broadening. Finally, Borra et al. (1983) found no circular polarization in 4 CP4 stars with $\mathrm{P}-\mathrm{Ga}$ anomalies, although with fairly large uncertainties $(500-800 \mathrm{G})$.

Generally speaking, there are indeed two quite different sequences of $\mathrm{CP}$ stars, in regard of their magnetic field properties. Large-scale organized fields are present only in the CP2 and in some CP4 stars. The status for the "nonmagnetic" sequence is on the other hand much less clear. The probable detection of a field with a complex structure in a few Am stars does not mean that it is a general property, although Takeda (1991) predicted that small relative intensifications of the pair of Fe II lines (actually even negative values, i.e. $W(6149.2)>W(6147.7))$ might be an indication of a field. However, one should stress that there is presently no detection of a CP1 or CP3 stars with a strong field. All CP stars with resolved magnetically split lines are CP2 stars, and no sharp-lined CP1/CP3 stars exhibit such resolved lines. An effort to settle the question of possible complex fields of weak or moderate strengths in CP1/CP3 stars is therefore needed. 


\section{MAGNETIC FIELDS AND SPOTS ON THE STELLAR SURFACE}

A main characteristic of the magnetic CP2 and CP4 stars is their periodic light and spectrum variations. These variations are due to the inhomogeneous distribution of chemical elements over the stellar surface, modulated by the rotation. The structure of the magnetic field is still ill-known (it may even not retain a cylindrical symmetry, see Mathys, these proceedings), but at least the spherical symmetry must break down. In some ways, the distribution of the chemical elements over the stellar surface has to be related to the field structure. Even a weak field appears to be very effective to destroy the spherical symmetry, as shown by $\epsilon$ UMa. As mentioned above, its field strength is of the order of $100 \mathrm{G}$, but this star show very strong spectral variations, which translates to high abundance contrasts over the surface (Rice \& Wehlau 1991). Donati (1990) showed the good match between the magnetic field geometry and the maps for chromium and iron. One may therefore want to use the distribution of some chemical elements over the stellar surface as a probe of the field structure. This link has still to be more firmly established, since some stars appear to show more patchy structures, which might imply a field structure more complex than a simple dipole. It may seem straightforward, but still reasonable, to suggest that these light and spectrum variations may at least give an indirect indication of the presence of a magnetic field in these stars. However, due to line-of-sight effects, one can probably not directly relate statistical variability properties to the distribution of magnetic field strengths.

On the other hand, stars having a magnetic field with a complex structure would probably have also a complex distribution of the chemical elements over their surface. Despite several claims in the literature, the spectrum and light variability of CP1 and CP3 stars is still ill-assessed. If some CP1 stars, and possibly some CP3 stars, have small-scale structures over their surface, it is much more probable that the spectrum variations would be barely or not directly detectable. However, even without variability, such structures can be detected, as first proposed by Khokhlova (1982). She pointed out that the non-linear character of the curve-of-growth leads to derive different values of a "mean" abundance from spectral lines of different strengths for a star with a spotted surface. This disparity may thus serve as a tool for identifying chemical inhomogeneities on stars which does not show any spectral variations, as long as all other aspects of the abundance analysis are well in hand.

I have recently reinvestigated the effects of stellar surface inhomogeneities on the curve-of-growth with a simple model. Let us assume that the structure on the surface is complex enough, so that there are numerous little patches randomly distributed over the surface. These spots have a different abundance for a given element, characterized by the abundance ratio relative to the background abundance. The same model atmosphere is adopted to calculate the radiative transfer inside and outside the spots (which may be questionable if the spots have large enhancements of elements like iron or silicon). With these assumptions, it is quite straightforward to compute the emergent flux, and then to build a curve-of-growth for different abundance contrasts and filling factors (relative total surface of the spots). Fig. 2 displays one case for a Ti II line in the blue spectrum, with an abundance 100 times larger in the spots and several filling factors, assuming a LTE model atmosphere with $T_{\text {eff }}=12000 \mathrm{~K}, \log g=4(\mathrm{Ku}$ rucz 1979) and no microturbulence. The largest differences occur at (and just 

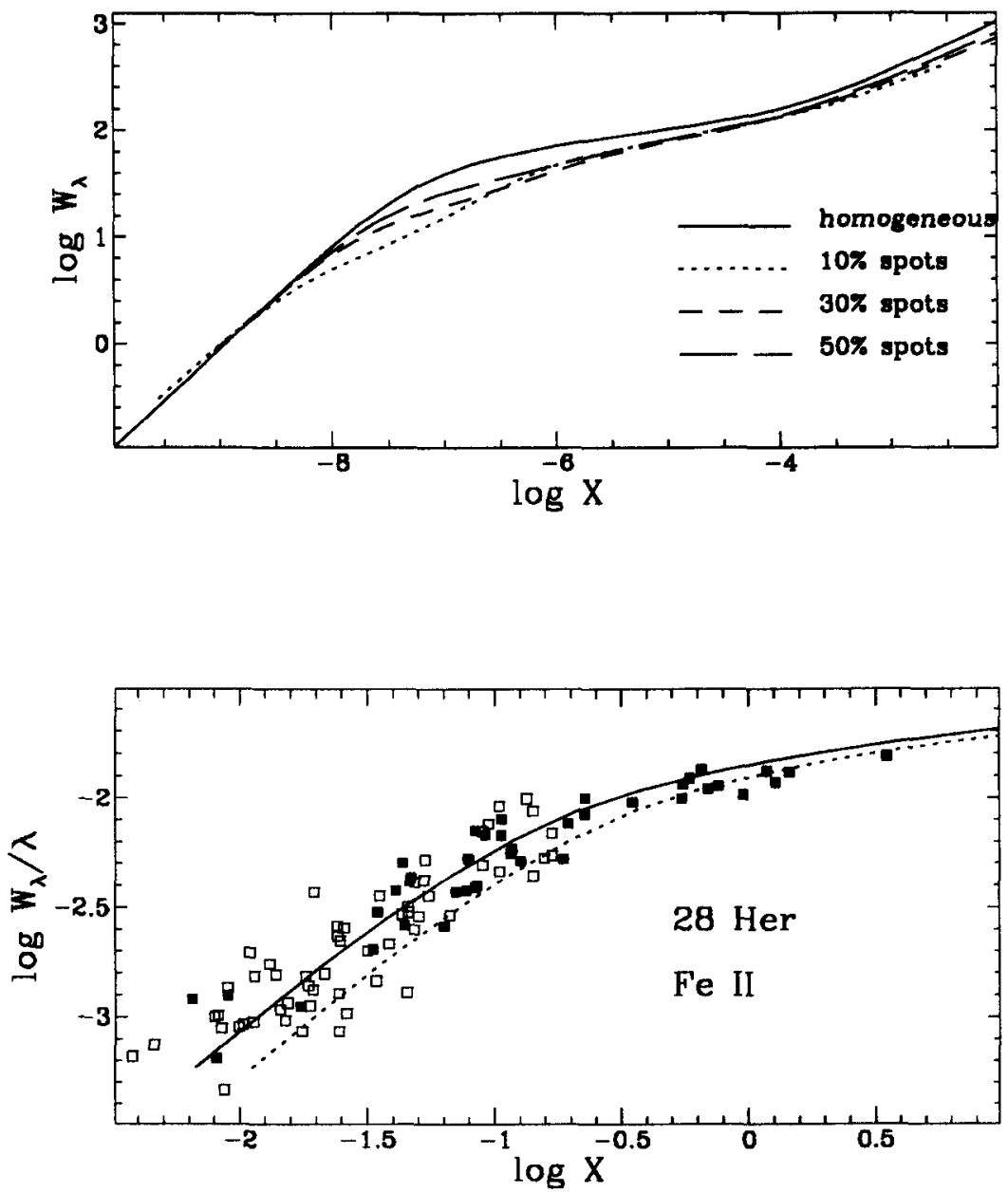

Fig. 2. The top pannel displays the theoretical curve-of-growths $\left(\xi_{t}=0\right.$ $\mathrm{km} / \mathrm{s}$ ) for an homogeneous photosphere (full line) and for spotted stars with various filling factors. The lower panel shows observed Fe II data for the CP3 star 28 Her with an appropriate curve-of-growth $\left(\xi_{t}=0 \mathrm{~km} / \mathrm{s}\right)$, fitting the weakest (full line) and the strongest Fe II lines (dotted line). Low ( $E_{\text {exc }}<3 \mathrm{eV}$, filled squares) and high excitation (empty squares) lines have been distinctly plotted.

before) the shoulder of the curve-of-growth, with smaller equivalent widths for the spotted models. If the background abundance is the same in models with and without metal-rich spots, the equivalent width is indeed larger in the model with spots. In Fig. 2, I have rather chosen to have the linear parts coincide to show the differences in the curve-of-growth shapes, which means that a given value of $\log X$ corresponds to different background abundances. The change in shape 
is quite straightforward to interpret: the line becomes saturated sooner in the metal-rich spots. It must be stressed that the curve-of-growths for spotted models lay below the curve-of-growth for an homogeneous model with $\xi_{t}=0 \mathrm{~km} / \mathrm{s}$, therefore in the opposite direction than the effect of the microturbulence!

The predicted differences at the shoulder of the curve-of-growth are fairly large (more than a factor 2 in $W_{\lambda}$ ), and might be discernible if the other parameters, like $g f$-values, are known with a sufficient accuracy. While $\mathrm{Ti}$ and Cr possibly show larger abundance contrasts than Fe on the surface of some CP2 stars, the accuracy of oscillator strengths for Fe II is indeed better and therefore offers better prospects. To test the prediction of the spotted model, the best case is a star which has a null microturbulence. Analyses of CP1 stars reveal high microturbulences, but several CP3 stars are good candidates. I have selected $28 \mathrm{Her}$, a $\mathrm{B} 9 \mathrm{p} \mathrm{MnHgSr}$ star, which have been recently analyzed by Adelman (1988b). Fig. 2 displays an empirical curve-of-growth, built from his equivalent widths measurements and the listed $g f$-values. A theoretical curve-ofgrowth $\left(\xi_{t}=0 \mathrm{~km} / \mathrm{s}\right)$ is fitted through the weak lines, and the same abundance is deduced using the lines with $5 \leq W_{\lambda} \leq 20 \mathrm{m \AA}$ and with $10 \leq W_{\lambda} \leq 35 \mathrm{~m} \AA$. On the other hand, the stronger lines $\bar{W}_{\lambda} \geq 45 \mathrm{~m} \AA$ are systematically below the curve-of-growth, as would predict a model with spots. The corresponding abundance discrepancy is about 0.22 dex. How significant is this result? The equivalent widths seem to be fairly accurate (good quality observations, sharplined spectrum). The scatter around the curve-of-growth is 0.17 dex, and may probably be accounted mostly by the quality of the $g f$-values. In this respect, the discrepancy is barely larger than the scatter, but it is a systematic shift! Preliminary explorative results for the Ti II lines in few other stars point in the same direction. A strong case cannot be made at the present stage, but I would like to stress how important it is to improve the quality of the atomic data. With a set of $g f$-values accurate at the $1 \%$ level (i.e. a similar accuracy than in present astrophysical data), we would be able to study any remaining scatter or departures from the curve-of-growth to get more insights on these stellar atmospheres, like departures from LTE, vertical stratification of the chemical elements, detection of complex structures on the stellar surface, adequacy of the model atmospheres.

\section{ABUNDANCE SCATTER}

Beyond the magnetic field, the main driving parameter for chemical anomalies seems to be the effective temperature: the CP3 stars appear in a hotter range than the CP1 stars, and similarly for the ApSi stars relative to the ApSrCrEu stars. Inside each of these groups, there is a large scatter on the observed abundance anomalies, occasionally larger than 1 dex. However, it is sometimes possible to point out definite trends with the effective temperature. In particular, Adelman (1988b, 1989) found a correlation of the Si and the $\mathrm{Mn}$ abundances with $T_{\text {eff }}$ for CP3 stars, but many correlations found between abundances and stellar parameters in previous studies were not confirmed. Adelman (1988b) noted how important it is to determine accurately the stellar parameters, because CP3 stars with similar effective temperatures and surface gravities should have similar abundances if there are no other hidden variables. I would like to stress here this point, underlying that simple correlations with $T_{\text {eff }}$ only may possibly 


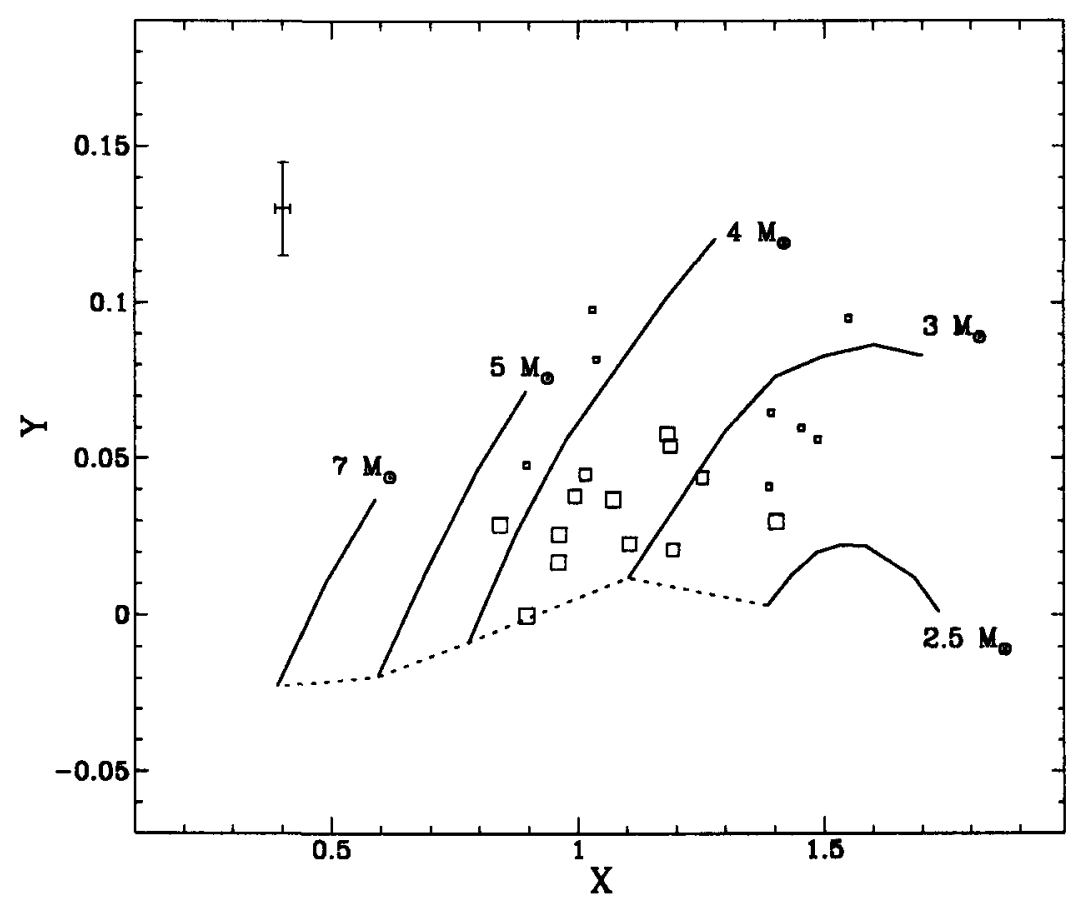

Fig. 3. CP3 stars in the $Y$ vs $X$ diagram of the Geneva photometric system, with evolutionary tracks and a ZAMS (dotted line). The size of the points are related to the gallium abundance. An estimate of uncertainties is indicated by the errorbars in the upper left corner.

be physically misleading, though statistically real. Stars with the same effective temperature may indeed have a different mass, and therefore a different age. If the surface chemical anomalies change during the main-sequence evolution, we would expect then some apparent scatter of the observed abundances inside the CP star groups. Uncertainties on the distances and the luminosities of field stars make difficult the use of the HR diagram, but some progress was achieved during the last years to derive more accurate surface gravities. North (these proceedings) discuss the case of CP stars in clusters.

A recent recalibration of the Geneva photometry in terms of $T_{\text {eff }}, \log g$ and mass for B stars (North \& Nicolet 1990) can be applied to CP3 stars to derive the stellar parameters. Their colours are not affected by the chemical peculiarity, contrary to the case of the CP2 stars. Subgroups with small mass intervals have been set up, and abundance changes are looked for inside these subgroups (the cooler stars being in this case the older stars). Lanz et al. (1993) recently completed a study on gallium in CP stars, and they have followed this approach for the CP3 stars. Gallium is an interesting element to study regarding the question of secular changes in the surface abundance, because the large $\mathrm{Ga}$ overabundances are indeed one typical peculiarity of the CP3 stars, although it is observed only in about half of the CP3 stars! Fig. 3 displays the CP3 stars in the $X-Y$ diagram of the Geneva photometric system, selecting the 
stars studied by Takada-Hidai et al. (1986). These two photometric parameters have been built to be insensitive to interstellar reddening (at least for moderate reddenings and for the normal absorption law), and are related primarily to the effective temperature for $X$ and to the luminosity for $Y$. The evolutionary tracks have been calculated by Maeder \& Meynet (1988) and translated to the $X-Y$ diagram by North \& Nicolet (1990); only the main-sequence stages are shown. Despite large uncertainties, it clearly appears that the stars without detected gallium lines (the smallest squares in Fig. 3) are the most evolved stars, while the only star (HD 358) possibly very close to the Zero Age Main Sequence is the star with the highest gallium abundance. The stars with a mass close to $3.5 M_{\odot}$ may suggest that the gallium overabundance already decreases during the first stages. Lanz et al. (1993) are therefore proposing that the surface overabundance of gallium in the CP3 stars may disappear in time-scales of about $1-210^{8}$ years, explaining why some CP3 stars are not gallium-rich.

This case points out that the evolutionary effects may be regarded as one potential reason for the large diversity of chemical anomalies in CP stars. Similar indications for other elements would be very important. A next step beyond the classification scheme proposed by Preston (1974) to enlighten the similarities and the differences between the CP stars should be a multivariate statistical approach trying to relate the abundances of key elements to stellar parameters (mass, age, rotation, magnetic field strength). Direct correlations might give first hints, but obviously they have not been successful to achieve a better description. First steps were made by Cowley \& Henry (1979) and Cowley (1983), who applied a cluster analysis technique to a sample characterized by the spectral strength (WCS parameter) of iron-peak and lanthanides elements. They found a different classification from these two groups of chemical elements; moreover, the rare-earth patterns of some magnetic CP2 stars are more closely related to the patterns in the CP1 stars than to those in other magnetic CP2 stars. Cowley (1983) also pointed out the similarities between peculiar late-type stars and some CP stars. Such techniques appear to be full of promises, if a sufficiently large sample with homogeneous and accurate abundances can be set up. This sample has to contain about 50 to 100 stars of different groups, with the stellar parameters and the abundances for elements very sensitive to various physical processes like the radiative diffusion, the $r$ - and the s-process, the gas-dust differentiation in proto- or circum-stellar material. Unfortunately, such a sample cannot (by far) be set up from the literature (see e.g. the status for CP3 stars reported by Takada-Hidai 1991), but an actual understanding of the similarities and the differences between the CP stars, and of the physical processes at work, justifies fully an renewed effort to gather these data.

\section{CONCLUSIONS}

Strong magnetic fields with a large-scale organization are only present in CP2 stars, which are therefore definitely different of the CP1 and the CP3 stars in this regard. But it does not exclude that these stars are more closely related to magnetic CP stars than previously assumed, if the recent findings of magnetic fields with a complex structure turn to be a more general characteristics of the so-called "non-magnetic" stars. The large differences in chemical abundances may be better understood from a multivariate approach relating the abundances 
to all the characteristic stellar parameters (mass, age, magnetic field, angular momentum) to unveil the working physical processes and their secular evolution.

Acknowledgements: The search of magnetic fields in Am stars is carried on in collaboration with G. Mathys. I would like to thank also W. Wehlau for discussions on "spotted" stars, and I. Hubeny for a careful reading of the manuscript.

\section{REFERENCES}

Adelman S.J., Cowley C.R., 1986, In: Upper Main Sequence Stars with Anomalous Abundances, Proc. IAU Coll. 90, Cowley C.R., Dworetsky M.M., Mégessier C. (eds), Reidel, Dordrecht, p. 305.

Adelman S.J., 1988a, MNRAS 235, 749.

Adelman S.J., 1988b, MNRAS 235, 763.

Adelman S.J., 1989, MNRAS 239, 487.

Babcock H.W., 1947, ApJ 105, 105.

Babcock H.W., 1958, ApJS 3, 141.

Bohlender D.A., Landstreet J.D., 1990, ApJ 358, L25.

Borra E.F., Landstreet J.D., 1980, ApJS 42, 421.

Borra E.F., Landstreet J.D., Thompson I.B., 1983, ApJS 53, 151.

Cayrel R., Burkhart C., Van't Veer C., 1991, In: Evolution of Stars: the Photospheric Abundance Connection, Proc. IAU Symp. 145, Michaud G. \& Tutukov A. (eds), Kluwer, Dordrecht, p. 99.

Conti P., 1969, ApJ 156, 661.

Cowley C.R., 1983, In: Statistical Methods in Astronomy, ESA-SP 201, p. 153.

Cowley C.R., Henry R., 1979, ApJ 233, 633.

Donati J.-F., 1990, PhD Thesis, University Paris VII.

Khokhlova V.L., 1982, Sov. Astron. Lett. 8, 163.

Kurucz R.L., 1979, ApJS 40, 1.

Landstreet J.D., 1982, ApJ 258, 639.

Landstreet J.D., 1992, A\&A Rev. 4, 35.

Lanz T., Artru M.-C., Didelon P., Mathys G., 1993, A\&A (in press).

Lanz T., Mathys G., 1992, to be submitted to A\&A.

Mathys G., 1988, In: Elemental Abundance Analyses, Adelman S.J. \& Lanz T. (eds), Inst. Astron. Univ. Lausanne, p. 101.

Maeder A., Meynet G., 1988, A\&AS 76, 411.

Mathys G., 1989, Fund. Cos. Phys. 13, 143.

Mathys G., 1990, A\&A 232, 151.

Mathys G., Lanz T., 1990, A\&A 230, L21.

Mathys G., Lanz T., 1992, A\&A 256, 169.

North P., Nicolet B., 1990, A\&A 228, 78.

Preston G.W., 1971, ApJ 164, 309.

Preston G.W., 1974, Ann. Rev. A\&A 12, 257.

Rao S.S., Abhyankar K.D., Nagar P., 1990, ApJ 365, 336.

Rice J.B., Wehlau W.H., 1991, A\&A 246, 195.

Ryabchikova T.A., 1991, In: Evolution of Stars: the Photospheric Abundance Connection, Proc. IAU Symp. 145, Michaud G. \& Tutukov A. (eds), Kluwer, Dordrecht, p. 149. 
Takada-Hidai M., 1991, In: Evolution of Stars: the Photospheric Abundance Connection, Proc. IAU Symp. 145, Michaud G. \& Tutukov A. (eds), Kluwer, Dordrecht, p. 137.

Takada-Hidai M., Jugaku J., 1992, PASP 104, 106.

Takada-Hidai M., Sadakane K., Jugaku J., 1986, ApJ 304, 425.

Takeda Y., 1991, PASJ 43, 823.

Wolff S.C., 1983, The A Stars: Problems and Perspectives, NASA-SP 463. 